\title{
A COMPARISON OF THE EFFICACY OF HOLMIUM LASER SCLEROSTOMY AB EXTERNO VERSUS TRABECULECTOMY IN THE TREATMENT OF GLAUCOMA
}

\author{
FRANK G. AH-FAT and CHRISTOPHER R. CANNING \\ Southampton
}

\section{SUMMARY}

We compared 15 patients who had undergone Holmium laser sclerostomy ab externo with 15 who had had trabeculectomy. In the short term, laser sclerostomy led to adequate control of intraocular pressure, but in the longer term it compared unfavourably with trabeculectomy in terms of efficacy, complications and reoperation rate. At 1 year follow-up, 8 patients in the laser group had had to undergo a second operation compared with none in the control trabeculectomy group, and 7 were still on glaucoma medication compared with 2 in the control group. Iris prolapse into the internal sclerostomy ostium within 2 months accounted for most failures, and was only partially amenable to Nd:YAG peripheral iridectomy. This common complication seems to be related to anterior chamber depth. There also appears to be a tendency for blockage of the sclerostomy with cellular or fibrinous debris. Recent literature is reviewed and modifying strategies discussed.

In the last two decades, trabeculectomy has become widely accepted as the standard surgical treatment for glaucoma. It has proved itself to be a safe, reliable and highly reproducible procedure. In recent years, however, there has been a surge of interest concerning the use of lasers as an alternative in performing fistulising procedures. Laser sclerostomy offers the advantage of being a faster, simpler and less invasive procedure that can be carried out on an out-patient basis and with a potentially shorter overall patient rehabilitation. An ab interno endoscopic approach eliminates the need for conjunctival dissection, the main factor in bleb fibrosis and late failure, but it involves anterior chamber manipulation. While a gonioscopic delivery of laser energy overcomes this problem, it

Correspondence to: Dr Frank G. Ah-Fat, MB ChB, Southampton Eye Unit, Southampton General Hospital, Tremona Road, Southampton SO16 6YD, UK. tends to be limited by technical difficulties. ${ }^{1}$ An ab externo approach with specially designed probes, on the other hand, can help to reduce the extent of conjunctival dissection and keep the procedure quick and minimally invasive. THC:YAG ('Holmium') laser sclerostomy ab externo is the best known of the laser sclerostomies and recent reports have been encouraging. In this paper we analyse our early experience with Holmium laser sclerostomy $a b$ externo and compare its outcome with that of standard trabeculectomy.

\section{MATERIALS AND METHODS}

\section{Patient Selection}

Fifteen patients who had undergone Holmium laser sclerostomy ab externo with a minimum of 1 year follow-up were matched retrospectively for age, sex, diagnosis and treatment with 15 who had undergone standard trabeculectomy during the same period (Table I). Their ages ranged from 29 to 85 years (mean 72.4 years). Twentyeight patients suffered from chronic open angle glaucoma and 2 from angle recession glaucoma. Their average preoperative intraocular pressures at the time of listing for surgery measured $25.6 \mathrm{mmHg}$ in the Holmium laser group and $26.5 \mathrm{mmHg}$ in the trabeculectomy group (Table II). Surgery was performed for uncontrolled intraocular pressures despite maximum medical treatment, with an average of 1.9 medications in the Holmium group and 2.1 in the trabeculectomy group (Table III). None of the patients had had previous surgery in the eye concerned.

\section{Surgical Technique}

All procedures were performed on an in-patient basis. Local anaesthesia was achieved with a retrobulbar injection of $3-5 \mathrm{ml}$ of a $1: 1$ mixture of $2 \%$ lignocaine and $0.75 \%$ bupivacaine. Through a $1 \mathrm{~mm}$ incision $12 \mathrm{~mm}$ 
Table I. Diagnosis and age match of the Holmium sclerostomy and control trabeculectomy groups

\begin{tabular}{lccccc}
\hline & \multicolumn{2}{c}{ Diagnosis } & & & Age \\
\cline { 2 - 5 } \cline { 5 - 6 } & $\begin{array}{c}\text { Chronic open } \\
\text { angle glaucoma }\end{array}$ & $\begin{array}{c}\text { Angle recession } \\
\text { glaucoma }\end{array}$ & & Mean & Range \\
\hline Holmium laser $(n=15)$ & 14 & 1 & 72 & $29-85$ \\
Control trabeculectomy $(n=15)$ & 14 & 1 & 73 & $33-82$ \\
\hline
\end{tabular}

Table II. Comparison of mean pre-operative and post-operative intraocular pressures in the laser and control groups

\begin{tabular}{|c|c|c|c|c|c|c|}
\hline & \multirow{2}{*}{$\begin{array}{l}\text { Pre-operative } \\
\text { intraocular } \\
\text { pressures } \\
(\mathrm{mmHg})\end{array}$} & \multicolumn{5}{|c|}{ Post-operative intraocular pressures $(\mathrm{mmHg}$ ) } \\
\hline & & 1 day & 1 week & 1 month & 3 months & 12 months \\
\hline Holmium laser group $(n=15)$ & $25.6 \pm 6.4$ & $11.4 \pm 6.0$ & $18.5 \pm 6.5$ & $25.2 \pm 10.4$ & $17.5 \pm 8.6$ & $14.1 \pm 6.7$ \\
\hline $\begin{array}{l}\text { Trabeculectomy group }(n=15) \\
p \text { value }\end{array}$ & $\begin{array}{c}26.5 \pm 5.9 \\
\mathrm{NS}\end{array}$ & $\begin{array}{c}8.8 \pm 4.2 \\
\mathrm{NS}\end{array}$ & $\begin{array}{l}10.8 \pm 4.5 \\
<0.01\end{array}$ & $\begin{array}{c}14.6 \pm 7.5 \\
<0.01\end{array}$ & $\begin{array}{c}15.6 \pm 8.0 \\
\mathrm{NS}\end{array}$ & $\begin{array}{l}12.9 \pm 7.0 \\
\mathrm{NS}\end{array}$ \\
\hline
\end{tabular}

$p$ values were calculated by student's $t$-tests (NS, not significant).

away from the limbus, a 22 gauge $(712 \mu \mathrm{m})$ Holmium laser probe was tunnelled subconjunctivally towards the limit of the conjunctival insertion. The energy level was set at $90-120 \mathrm{~mJ}$ at a pulse rate of 5 per second. The tip was held tangentially with the helium-neon beam aimed away from the iris plane and roughly towards the centre of the anterior chamber. Between 18 and 39 pulses were required, with successful formation of the sclerostomy signalled by the appearance of small gas bubbles and release of debris into the anterior chamber. The conjunctiva was closed with a single absorbable suture.

All the patients in the control group underwent standard trabeculectomy under retrobulbar anaesthesia with a fornix-based flap.

\section{Follow-up}

All glaucoma medications were initially stopped after surgery. Routine follow-up was at 1 day, 1 week, 1 month, 3 months, 6 months and 1 year.

\section{RESULTS}

We found the technique of Holmium laser sclerostomy $a b$ externo simple and straightforward, requiring less than 5 minutes of operating time. There were no complications in the intra-operative or immediate post-operative period in either group. All patients had adequate bleb formation with no iris prolapse or peaked pupil following withdrawal of the laser probe. On the first post-operative day, 1 patient in the Holmium laser group developed a hyphaema which cleared rapidly. The mean pressure was $11.4 \mathrm{mmHg}$, with 13 eyes showing excellent control. This was comparable with the standard trabeculectomy group (mean pressure of $8.8 \mathrm{mmHg} ; p$ value not significant) (Table II).

Table III. Comparison of mean number of glaucoma medications
In the intermediate and longer term, Holmium laser sclerostomy ab externo compared unfavourably with standard trabeculectomy. There was a rise in pressure with gradual shrinkage of the bleb in all but 3 eyes in the laser group within the first 2 months. Failure at this stage would normally be expected to be caused by sclerostomy failure rather than bleb fibrosis, and the complications encountered are as summarised in Table IV but were of two main types:

1. Iris prolapse into the internal sclerostomy ostium. This was manifest by peaking of the pupil and was confirmed by gonioscopy in 8 patients. One was noted on the first post-operative day, and the remainder between 5 days and 2 months. Nd:YAG laser peripheral iridectomy was attempted and this was sufficient to free the iris and lower the intraocular pressure in 5 patients $(63 \%)$, while the remainder eventually required trabeculectomy. However, despite an initially successful and patent peripheral iridectomy, there was a tendency either for the iris to prolapse ( 3 patients) or for the pressure to rise again (2 patients), possibly due to blockage by iris debris and pigment. Trabeculectomy was eventually performed in a further 3 patients.

2. Sclerostomy blockage by cellular or fibrinous material. In 4 patients the internal sclerostomy ostium was clearly visible on gonioscopy, but the intraocular pressure was poorly controlled. We presumed this was caused by sclerostomy blockage by cellular or fibrinous material, and we performed ocular massage. In 2 patients intraocular pressure was controlled with topical medication, but 2 further patients underwent trabeculectomy within 3 months.

Trabeculectomy was performed with the scleral flap

\begin{tabular}{lccccc}
\hline & & \multicolumn{3}{c}{ Post-operative } & \\
\cline { 3 - 6 } & Pre-operative & 1 week & 1 month & 3 months & 12 months \\
\hline Holmium laser $(n=15)$ & $1.9 \pm 0.4$ & 0 & $0.57 \pm 0.3$ & $0.79 \pm 0.5$ & $1.07 \pm 0.6$ \\
Trabeculectomy $(n=15)$ & $2.1 \pm 0.6$ & 0 & 0 & $0.13 \pm 0.1$ & $0.2 \pm 0.1$ \\
$p$ value & $\mathrm{NS}$ & $\mathrm{NS}$ & $<0.01$ & $<0.01$ & $<0.01$ \\
\hline
\end{tabular}


Table IV. Complications and outcome of Holmium laser sclerostomy in 15 patients

\begin{tabular}{lcll}
\hline Complications & $\begin{array}{c}\text { No. of } \\
\text { patients }\end{array}$ & Treatment \\
\hline Raised pressure, with visibly free internal ostium & 4 & Massage and topical treatment (2); trabeculectomy (2) \\
Iris prolapse & 8 & Nd:YAG peripheral iridectomy (8) \\
Failure to free iris with Nd:YAG peripheral iridectomy & 3 & Trabeculectomy (1); trabeculectomy + topical treatment (2) \\
Recurrent iris prolapse despite Nd:YAG peripheral iridectomy & 3 & Trabeculectomy (2); trabeculectomy + topical treatment (1) \\
Presumed blockage by debris after Nd:YAG peripheral iridectomy & 2 & Ocular massage + topical treatment (2) \\
\hline
\end{tabular}

adjacent to the previous sclerostomy, and this was uneventful except for 1 case which developed a persistent aqueous leak and required resuturing.

At 1 year follow-up the mean intraocular pressure in the Holmium laser group was $14.1 \mathrm{mmHg}$, with 8 patients having required trabeculectomy and 7 still needing topical medication, including 3 who had undergone a second operation (mean number of medications: 1.07) (Table III). In comparison, the control standard trabeculectomy group showed fewer complications, as reflected by fewer outpatient visits in the first 3 months and better pressure control. There were 6 cases of post-operative hyphaema with no serious sequelae (Table V). None of the patients in the control group developed iris prolapse or required a second operation and only 2 were still on topical medication (mean 0.2 ) after 1 year. The mean intraocular pressure was $12.9 \mathrm{mmHg}$.

\section{DISCUSSION}

Holmium laser sclerostomy compared unfavourably with standard trabeculectomy. Only 3 of the 15 patients (i.e. $20 \%$ ) in the Holmium sclerostomy group achieved stable pressures without any medication or further surgical intervention at 1 year follow-up (i.e. desired success) compared with 13 in the trabeculectomy group (87\%) (Table VI). In a further 4 laser patients, adequate control was achieved through topical medication, giving a qualified success rate of $47 \%$. Failure was caused predominantly by iris prolapse leading to blockage of the fistula tract (53\%). $\mathrm{Nd}$ :YAG laser peripheral iridectomy was met with limited success. We suspect that a significant number of failures were also caused by sclerostomy blockage with cellular or inflammatory debris from the Holmium or Nd:YAG laser.

In the initial studies on laser sclerostomy, ab interno or ab externo, involving animal eyes, blockage of the fistula by iris tissue prolapsing or by fibrin or cellular debris was a common complication in the early and intermediate term..$^{2-4}$ There have been varying reports of closure of sclerostomy by iris tissue in procedures performed on glaucoma patients. In an earlier series of 26 episodes of Holmium laser thermal sclerostomy ab externo studied by Hoskins et al. ${ }^{5}$ iris incarceration was seen in all but 1 phakic patient but was a rare event in aphakic or pseudophakic patients. Intracameral injection of viscoelastic material was used to prevent this complication. Per-operative iris prolapse was also treated with limbal massage, but at post-operative follow-up, iris prolapse was almost the rule. In a later series of 49 procedures with 1 year follow-up, iris prolapse was noted in 9 of 22 phakic eyes. While laser peripheral iridectomy was initially successful, a significant number of patients still required topical medication for adequate control. One case of recurrent iris prolapse was treated with long-term topical dilating drops. ${ }^{6}$ The use of long-term pilocarpine on the other hand, does not necessarily prevent or reverse iris prolapse. McAllister and Watts $^{7}$ reported a $46 \%$ incidence of iris prolapse in a series of 30 patients, with $80 \%$ successfully dealt with using the $\mathrm{Nd}$ :YAG laser, but wondered whether iris tissue might still be left in the sclerostomy after the procedure. With an average follow-up of 7.5 months, the mean number of medications fell from 2.2 pre-operatively to 0.8 post-operatively.

Iris prolapse is rare in aphakic or pseudophakic eyes. An ab interno approach in aphakes and pseudophakes by Wilson and Javitt ${ }^{8}$ and $\mathrm{Nd}$ :YAG laser gave no report of iris prolapse. Kendrick and Kollarits' described a technique of combined cataract extraction with laser sclerostomy $a b$ interno in 15 eyes and reported no cases of iris prolapse. It is likely that the deepening of the anterior chamber following cataract extraction with or without intraocular implant is an important factor in reducing the chances of the peripheral iris plugging the internal sclerostomy ostium, and we would regard a narrow angle as a contraindication to laser sclerostomy. There also appears to be a learning curve in such procedures, especially with regard to the ability to fashion the sclerostomy well away from the iris plane while avoiding corneal burns or endothelial damage. Nonetheless, iris prolapse continues to remain a

Table V. Post-operative outcome and complications

\begin{tabular}{lcccc}
\hline & $\begin{array}{c}\text { Length of } \\
\text { hospital stay } \\
\text { (days) }\end{array}$ & $\begin{array}{c}\text { No. of outpatient } \\
\text { visits in the } \\
\text { first } 3 \text { months }\end{array}$ & Iris prolapse & $\begin{array}{c}\text { Topical medication } \\
\text { required for } \\
\text { pressure control }\end{array}$ \\
\hline Re-operations
\end{tabular}

${ }^{\text {a }} p$ value from Student's t-test.

${ }^{\mathrm{b}} p$ value from $\chi^{2}$-test. 
Table VI. Comparison of success rate of Holmium laser sclerostomy and trabeculectomy at 1 year follow-up

\begin{tabular}{lcc}
\hline & Desired success & Qualified success \\
\hline Holmium laser $(n=15)$ & $3(20 \%)$ & $7(47 \%)$ \\
Trabeculectomy $(n=15)$ & $13(87 \%)$ & $15(100 \%)$ \\
$p$ value $\left(\chi^{2}\right.$ test $)$ & $<0.01$ & $<0.01$ \\
\hline
\end{tabular}

Desired success, adequate pressure control $(<21 \mathrm{mmHg})$ without medication; qualified success, pressure controlled with or without medication

problem even in experienced hands. ${ }^{6}$ The use of intracameral viscoelastics helps to prevent per-operative iris incarceration, but renders the procedure more invasive and does not guard against late iris prolapse. Standard trabeculectomy relies on performing a sizeable peripheral iridectomy to prevent iris from blocking the fistula opening, and iris prolapse is rare. ${ }^{10}$ In laser sclerostomy, preoperative or post-operative laser iridectomy has been attempted with variable degrees of success in the short and long term. ${ }^{5-7}$ We believe this is explained by the limited size of the iridectomy that is feasible by laser as compared with surgical peripheral iridectomy. A pre-operative surgical iridectomy would, however, render the procedure lengthier and more invasive. It is also likely that plugging of the sclerostomy by iris debris may account for some failures.

Holmium laser sclerostomy ab externo offers the prospect of a quick, simple and minimally invasive procedure in the treatment of glaucoma. When compared with the gold standard of trabeculectomy, there was no significant difference in the length of hospital stay, and due to its simplicity it is often carried out on an out-patient basis. However, iris prolapse remains a vexing problem and is responsible for a significant number of failures. As an alternative to trabeculectomy, Holmium laser sclerostomy needs further evaluation with respect to efficacy and cost- effectiveness. Success in the long term may depend on careful patient selection, especially with regard to anterior chamber depth and accurate sclerostomy fashioning in the optimum plane away from the iris, in addition to meticulous post-operative follow-up and management of complications.

Key words: Holmium laser sclerostomy, Iris prolapse, Peripheral iridectomy, Trabeculectomy.

\section{REFERENCES}

1. Allan BDS, van Saarloos PP, Cooper RL, Constable IJ. Laser microsclerostomy for primary open angle glaucoma: a review of laser mechanisms and delivery systems. Eye 1992;6:257-66.

2. Javitt JC, O'Connor SS, Wilson RP, Federman JL. Laser sclerostomy $a b$ interno using a continuous wave Nd:YAG laser. Ophthalmic Surg 1989;20:552-6.

3. Park SB, Kim JC, Aquavella JV. Nd:YLF laser sclerostomy. Ophthalmic Surg 1993;24:118-20.

4. Wetzel W, Scheu M. Laser sclerostomy ab externo using mid infrared lasers. Ophthalmic Surg 1993;24:6-12.

5. Hoskins HD, Iwach AG, Vassiliadis A, Drake MV, Hennings DR. Subconjunctival THC:YAG laser thermal sclerostomy. Ophthalmology 1991;98:1394-400.

6. Iwach AG, Hoskins HD, Drake MV, Dickens CJ. Subconjunctival THC:YAG ('Holmium') laser thermal sclerostomy ab externo: a one-year report. Ophthalmology 1993;100:356-66.

7. McAllister JA, Watts PO. Holmium laser sclerostomy: a clinical study. Eye 1993;7:656-60.

8. Wilson RP, Javitt JC. Ab interno laser sclerostomy in aphakic patients with glaucoma and chronic inflammation. Am J Ophthalmol 1990;110:178-84.

9. Kendrick RM, Kollarits CR. Combined cataract-glaucoma surgery using the THC:YAG (Holmium) laser $a b$ interno without gonioscopy. Ophthalmic Surg 1992;23:697-9.

10. Cairns JE. Trabeculectomy: preliminary report of a new method. Am J Ophthalmol 1968;66:673-9. 